\title{
Influence of boundary conditions on the hydrodynamic forces of an oscillating sphere
}

\author{
Domenica Mirauda ${ }^{1, *}$, Marco Negri $^{2}$, Luca Martinelli ${ }^{2}$, and Stefano Malavasi ${ }^{2}$ \\ ${ }^{1}$ School of Engineering, Basilicata University, 85100 Potenza, Italy \\ ${ }^{2}$ Department of Civil and Environmental Engineering, Politecnico di Milano, 20133 Milano, Italy
}

\begin{abstract}
The design of submerged structures in sea currents presents certain problems that are not only connected to the shape of the obstacle but also to the number of acting forces as well as the correct modelling of the structures dynamic response. Currently, the common approach is that of integrated numerical modelling, which considers the contribution of both current and structure. The reliability of such an approach is better verified with experimental tests performed on models of simple geometry. On the basis of these considerations, the present work analyses the hydrodynamic forces acting on a sphere, which is characterised by a low mass ratio and damping. The sphere is immersed in a free surface flow and can oscillate along the streamwise and transverse flow direction. It is located at three different positions inside the current: close to the channel bottom, near the free surface and in the middle, and equally distant from both the bottom and free surface. The obtained results for different boundaries and flow kinematic conditions show a relevant influence of the free surface on the hydrodynamic forces along both the streamwise and transverse flow directions.
\end{abstract}

\section{Introduction}

Knowledge of the dynamic response of submerged and not-completely submerged structures in sea currents is fundamental for the correct design of a large number of off-shore structures (e.g., ocean risers, wind farms, marine turbines, oil conduits, platforms, buoys, power wave converters, and submerged floating tunnels). Although this topic has been considered in several previous studies, many questions remain unanswered. For example, in these types of structures, the modelling of the hydrodynamic response is usually subject to a linearization of the hydrodynamic force components considering: the inviscid flow, body subject only to conservative forces due to gravity and irrotational motion. Such linearization implies that the fluid oscillations are smaller than the wave length. As a consequence, the modelling of the hydrodynamic response of the oscillating structure does not allow us to consider the effects of refracted or steep waves or secondary, or of superior order, hydrodynamic effects. In addition, modelling situations in which the displacements of both submerged and floating bodies are larger than their typical dimensions is not allowed. Moreover, in most cases, these structures are modelled without considering the presence of free surface (waterair interface) effects, which therefore overlooks an important interference factor for body movements. In fact, this hypothesis can hide the effects of vortexinduced vibrations (VIV), which are not negligible whenever resonance or lock-in phenomena (where the frequency of VIV is close to the natural frequency of the submerged or floating system) occur. In fact, such phenomena are two of the most critical mechanical conditions for these structures.

Considering the complexity of the research topics and numerous different forces stressing the structure, integrated numerical modelling seems to be the most suitable method to consider the contributions of the most significant forces [1-3]. Additionally, the presence of a free surface influences hydrodynamic actions in a peculiar way, both in the case of a steady flow, such as a sea current, and unsteady ones, such as waves [4].

To assess their reliability, the resolving methods derived from integrated numerical modelling need to be compared with the results of the experimental analyses. Given the complexity of the phenomena, these involve models of simplified geometries (e.g., spheres, cylinders, and rectangles). In this complicated scenario, most studies in the literature focus on two-dimensional structures, such as rectangles and cylinders [5-7].

Few studies report the analysis of hydrodynamic forces acting on a three-dimensional body, such as a sphere [8-10]. In particular, Govardhan and Williamson [8] investigated the fluctuating transverse forces acting on an elastically mounted sphere that was constrained to move transversely to the flow direction. These forces were measured by force balance and utilising linear variable displacement transducers. The other two papers $[9,10]$ instead show a trend of the mean drag coefficient of tethered buoyant spheres that are free to oscillate

\footnotetext{
Corresponding author: domenica.mirauda@unibas.it
} 
along both the streamwise and transverse flow directions.

All of the above mentioned studies refer to spheres that are completely immersed in the current and distant from the free surface and channel bottom. In contrast to previous studies, this paper analyses the influence of both the free surface and channel bottom on the hydrodynamic forces acting on an elastically mounted sphere that is free to move in the streamwise $(x)$ and transverse $(y)$ flow directions.

\section{Experimental set-up}

The experimental set-up consisted of a deformable rodsphere system immersed in a steady flow and in a horizontal, non-tilted, Plexiglas open water channel with a rectangular cross section of $0.5 \times 0.6 \mathrm{~m}$ and a length of approximately $7 \mathrm{~m}$ (Fig. 1a). The sphere was composed of PVC, water filled, and with a diameter $D=0.087 \mathrm{~m}$ $[11,14]$. The surface of the sphere was covered with paint to reduce the surface roughness. A flexible cylindrical rod was used to retain the sphere and connect it to a fixed support structure.

The rod had a circular cross-section. The longer upper part was composed of Polyoxymethylene (Delrin) with a diameter $D_{l}=0.012 \mathrm{~m}$; the shorter lower part was composed of stainless steel with a much smaller crosssection and a diameter $D_{2}=0.003 \mathrm{~m}$ to avoid perturbing the flow in the proximity of the sphere.

A clutch linked the rod to a fixed support structure that was located above the channel (Fig. 1) and allowed the deformable length of the rod and the immersion of the sphere to change.

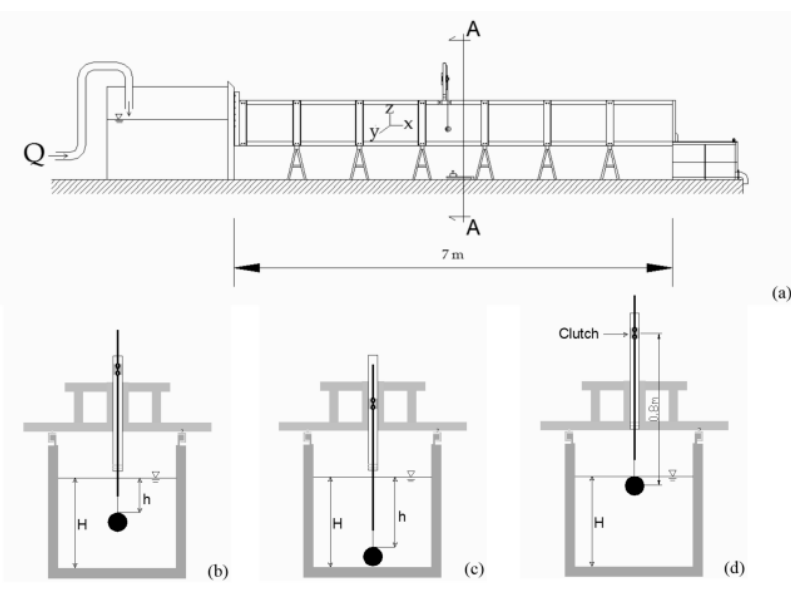

Fig. 1. Experimental channel; the transverse section of the channel and sphere positions at (b) $h^{*}=2$, (c) $h^{*}=3.97$, and (d) $\mathrm{h}^{*}=0$.

The rod flexibility allowed the sphere two degrees of freedom (in the streamwise, $x$, and transverse, $y$, directions).

The spherical body was immersed in a free surface flow with a water depth equal to 5 times the sphere diameter $(H=0.435 \mathrm{~m})$ to maintain the blockage coefficient $\left(\gamma_{b}=0.03\right.$, the ratio between the body frontal area and transverse section of the channel) constant. The flow had a constant turbulence level of approximately 10 $\%$, whereas the mean flow velocity, $U$, changed between 0.2 and $0.5 \mathrm{~m} / \mathrm{s}$.

Three different boundary conditions of the flow around the sphere were tested and were denoted by value $h^{*}$ of the ratio between $h$ (distance of the sphere upper surface from the free surface) and the sphere diameter, $D$. We considered a quasi-symmetric condition $(h *=2)$ with the sphere equally distant from the free surface and channel bottom (Fig. 1b) as well as two other conditions of asymmetric bounded flow (Figs. 1c and 1d): one with the sphere close to the channel bottom $\left(h^{*}=3.97\right)$ and the other with the sphere close to the free surface $\left(h^{*}=\right.$ $0)$.

The sphere displacements for the different flow velocities, $U$, were measured by image analysis through a Charge Coupled Device (CCD) camera, which allowed reconstruction of the sphere trajectories in the horizontal plane $(x-y)$. The camera, which was located below the water channel (Fig. 1a), permitted us to memorise the body $2 D$ displacements due to the presence of a marker placed below the sphere in-line with its barycentre [1114]. The time history of the $2 D$ movement of the sphere was provided by using a blob analysis algorithm to recognise the position, dimension and shape of the marker on each image. The sequence was pre-processed by a filter procedure. We set the level of the threshold filter at 100/255, which is a value that assured the negligible influence of the filtering on the calculated displacement. The high quality of the acquisitions and blob detection procedure used for the identification of the marker minimised the measurement uncertainty of the sphere displacements. This uncertainty was assumed to be less than the image's pixel dimension, which ranged between $0.4 \mathrm{~mm}$ and $0.5 \mathrm{~mm}$ (with an image resolution between 18.5 and $23 \mathrm{pixel} / \mathrm{cm}$ ). The position of the sphere on each frame was provided considering the barycentre of the marker, which had a mean area of approximately 100 pixel $^{2}$. Moreover, during the procedure of the displacement calculation, we controlled the dimension of the marker on the image so that it did not change more than $5 \%$ along the sequence [11-14].

\section{Dynamic characterisation of the rod- sphere system and determination of the hydrodynamic forces}

From a mechanical point of view, the composite rod acts as an elastic restraint for a sphere in the $x$ and $y$ directions. Furthermore, due to its small mass, its dynamics can be disregarded, and the fluid forces on the sphere can be simply computed by use of the dynamic equilibrium equation of a sphere that is considered to be a rigid body as well as by knowledge of its velocity, acceleration and forces exerted by the rod.

We assumed that the oscillations were of a small amplitude so that: (a) the forces exerted by the rod could be linearly related to the sphere displacement through a stiffness coefficient, $k$, and (b) the rod could also be linearly related to the velocity through a damping coefficient, $c$. The values of the spring stiffness, $k$, and 
damping coefficient, $c$, were determined from the modal properties estimated through dynamic experimentation in air as well as a parametric technique with reference to the geometry and structural boundary conditions of the experimental set-up described in Section 2. In particular, they were evaluated by disregarding the mass of the rod and by examining the motion of the sphere in the $y$ direction, assuming that the rod-sphere system behaves like a linear single degree-of-freedom (SDOF) oscillator of mass equal to that of a sphere filled with water $(m=$ $0.46 \mathrm{~kg}$ ). A comparison of the results with the theory of the SDOF oscillators permitted an assessment of the validity of the assumed linearity hypothesis for the rod forces at the displacement level involved by the testing in the water channel described in Section 2.

When using dynamic testing, it is easy and reliable to estimate the stiffness, $k$, if the mass is known, but damping is one of the most difficult modal structural properties to estimate. This is due to several reasons. One is the representation through viscous damping of different forms of dissipation, which can be due to several mechanisms of an internal or external nature, such as hysteretic damping, Coulomb-friction damping, velocity squared damping (hydrodynamic force), or other cinematic variables that are different from the velocity. Another reason is the measurement noise, which requires some form of averaging in the estimation process to eliminate its effects.

The classical method of the logarithmic decrement (Log-Dec) was adopted here to estimate the modal parameters of the rod-sphere system and was supplemented with averaging to mitigate the noise effects. The Log-Dec method can strictly only deal with SDOF systems, whereas in the case study, the length of the rod, its mass (albeit small) and the spatial distribution of the mass on the sphere only lead to an approximation of a SDOF oscillator, and therefore, more than one mode is possible. However, in practice, whenever the natural frequencies of the system are sufficiently separated, as in the case analysed here, the response of a SDOF oscillator can be obtained by isolating the single mode of interest utilising a band-pass filter. Care must be exerted in choosing the type of filter since it distort the signal in the time domain and cause bias error.

The adopted procedure is as follows:

a) recording of several free decay oscillations (Fig. 2) of the sphere were set free to oscillate from an initial position that is displaced in the flowtransverse direction, $y$, by $5 \mathrm{~cm}$ with respect to the static equilibrium position. A Brüel \& Kjær Type 4384 piezoelectric accelerometer with a sensitivity of $1.004 \mathrm{pC}^{-\mathrm{ms}^{-2}}$ at $159.2 \mathrm{~Hz}$ was used along with a 16 bits $\mathrm{ADC}$ at the sampling frequency of $500 \mathrm{~Hz}$;

b)estimate the power spectral density (PSD) function from the recorded signal using the Welch's method [15] and divide the input signal into eight overlapping segments with a $50 \%$ overlap (a Hamming window is used on each segment before estimating the PSD);

c)identify the PSD of the spectral bell and the damped natural frequency $\left(f_{d}=1.422 \mathrm{~Hz}\right)$ of the first mode in the air (Figs. 3 and 4). Due to the small value of damping, this frequency can be considered as that of the un-damped structure, $f_{n}$;

d)filter the free decay signal (Figs. 6 and 7) with a band-pass filter centred on $f_{n}$ to obtain the response of an SDOF (an order two band-pass Butterworth filter, Fig. 5, with lower and upper cut-off frequencies of $0.5 \mathrm{~Hz}$ and $2 \mathrm{~Hz}$, respectively, is applied to the time series first in the forward direction and then backwards to avoid phase distortion of the filtered signal);

e) separate the recorded signal into single episodes of free decay oscillations;

f) transform to the absolute value;

g)represent the results in a semi-log scale of each free decay oscillation (Figs. 8 and 9) and linearly interpolate the peaks to obtain the angular coefficient of the line, which when divided by $2 \pi f_{n}$, directly provides the value of the critic damping $\zeta=$ $c /\left[2(\mathrm{~km})^{0.5}\right]$.

Some tests were prematurely stopped (e.g., at $t=340 \mathrm{~s}$ in Fig. 2) due to the large coupling of the longitudinal $(x)$ and lateral $(y)$ vibration components. The non-perfect linear shape of the elastic rod induces this type of coupling and is also partially responsible for the deviation from linearity in the system response at low amplitudes, which is clearly shown by the scattering of the peaks in Figures 8 and 9 from $55 \mathrm{~s}$ onward. Also note that for the small values of acceleration at approximately $100 \mathrm{~s}$ shown in Figure 9, the accelerometer is close to its operational limits, and the effect of the signal to noise ratio may have contributed to the scattering in spite of the band-pass filtering.

This scattering can adversely influence the estimation of the damping factor value. Therefore, this was calculated for each single free decay episode; first, by using all of the peaks, and then, by subsequent confirmation using only the larger amplitude peaks corresponding to the first $20 \%$ of each signal, which are less affected by the effects of the rod geometric nonlinearity. The results are listed in Table 1. Nearly negligible differences were obtained from the two different estimates. A mean value of $\zeta=0.00443$ was finally selected from the results of the second column in Table 1.

Assuming a mass $m=0.46 \mathrm{~kg}$ from the natural frequency $f_{n}=1.422 \mathrm{~Hz}$ of the first mode, the stiffness and the damping coefficient (with $\zeta=0.00443$ ) in the air of the SDOF system can be computed as:

$$
\begin{aligned}
k & =\left(2 \pi f_{n}\right) 2 m=36.72 \mathrm{~kg} / \mathrm{s}^{2} \\
c & =2 \zeta(\mathrm{km})^{0.5}=0.036 \mathrm{~kg} / \mathrm{s}
\end{aligned}
$$

The values of $f_{n w}$ and $\zeta_{w}$ in water, which were equal to $1.213 \mathrm{~Hz}$ and 0.0038 , respectively, were obtained by multiplying the values of the same parameters in the air by the coefficient $\left[m^{*} /\left(m^{*}+C_{A}\right)\right]^{1 / 2}$ in which $m^{*}$ is the ratio between the system mass, $m$, and displaced fluid mass, $m_{d}\left(m^{*}=m / \pi \rho D^{3} / 6=1.34\right)$ and $C_{A}$ is the potential added mass coefficient $\left(C_{A}=0.5\right.$ for a sphere) when taking into account the water presence according to the paper by [8]. 


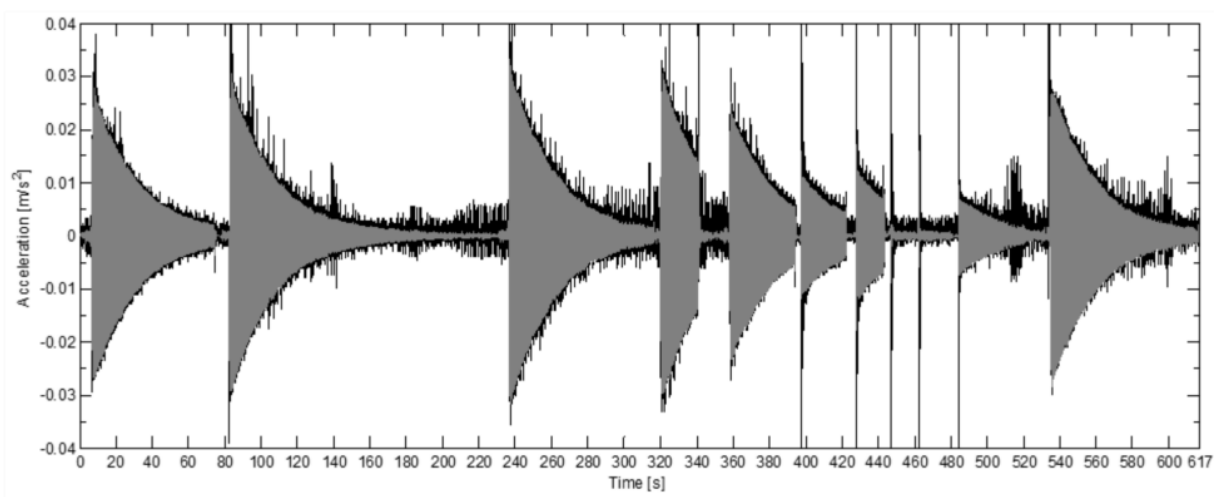

Fig. 2. The recorded acceleration signal showing several free decay episodes: black $=$ unfiltered, grey $=$ after filtering.
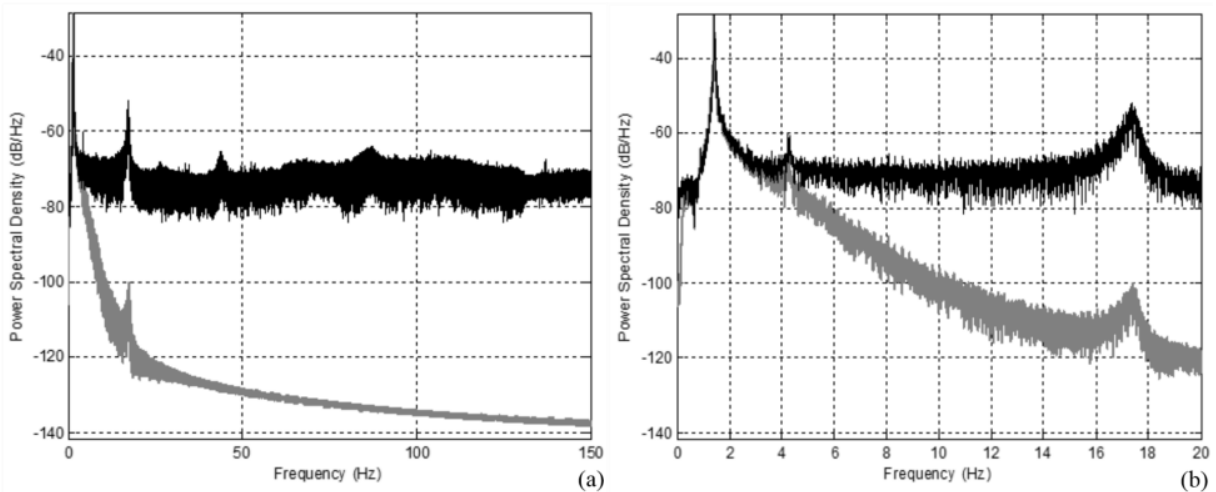

Fig. 3. Estimated power spectral density functions of accelerations: black $=$ unfiltered acceleration signal, grey $=$ after filtering. Overall view (a) and zoom-in (b).
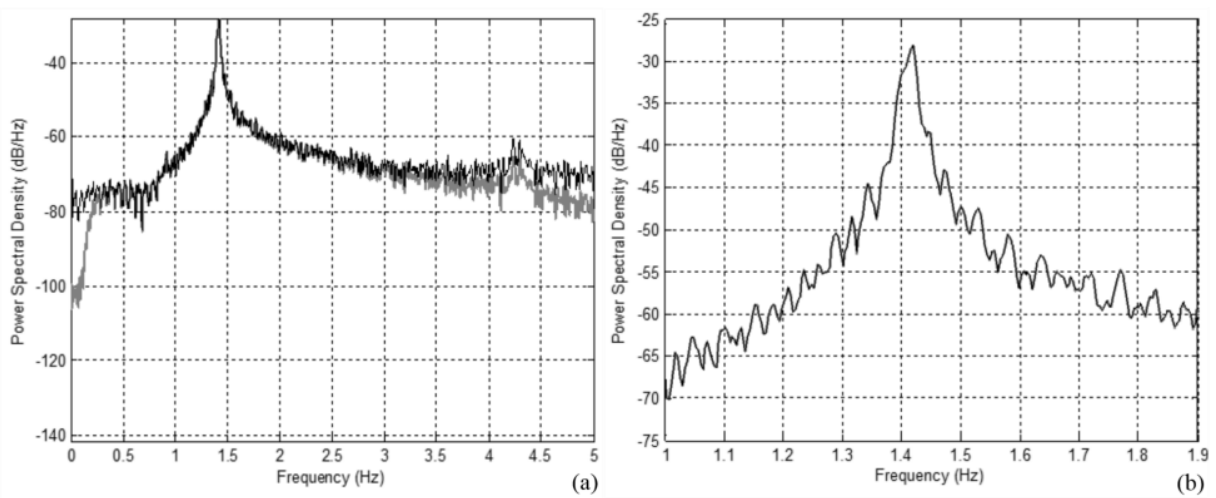

Fig. 4. Estimated power spectral density functions of accelerations: black $=$ unfiltered acceleration signal, grey $=$ after filtering. The larger zoom-in level ( $a$ and $b$ ) showing no attenuation of the spectral bell due to band-pass filtering.

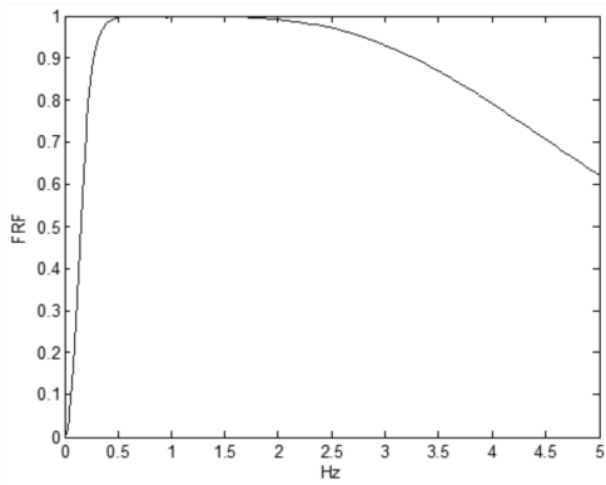

Fig. 5. The Frequency Response Function (FRF) of the adopted Butterworth 2nd- order filter.

\footnotetext{
* Corresponding author: domenica.mirauda@unibas.it
} 

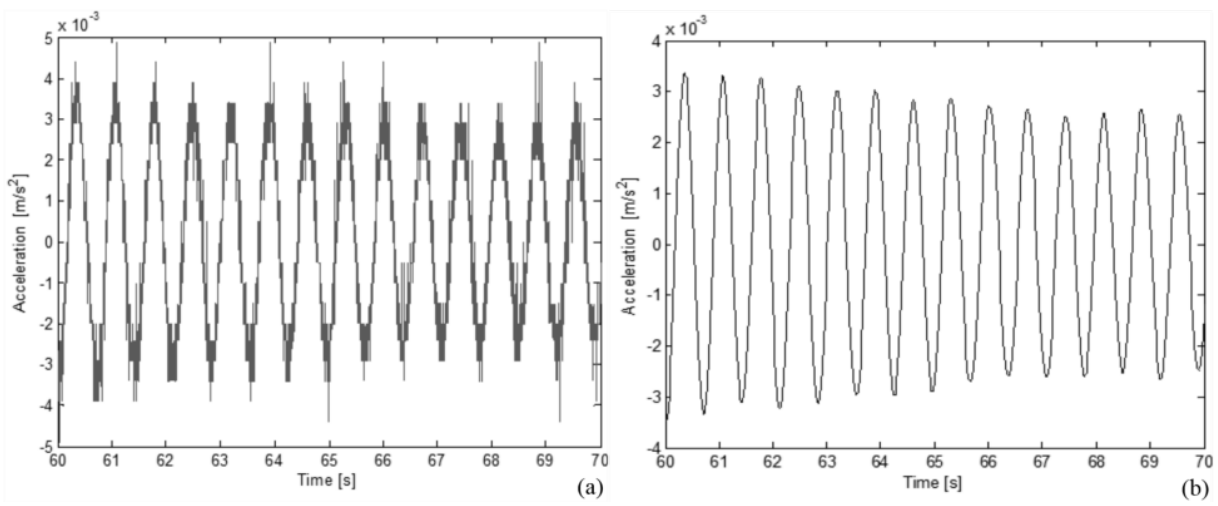

Fig. 6. The detail of the acceleration signal (a) before and (b) after filtering for the free decay oscillations episode at a time of 7-73 s.
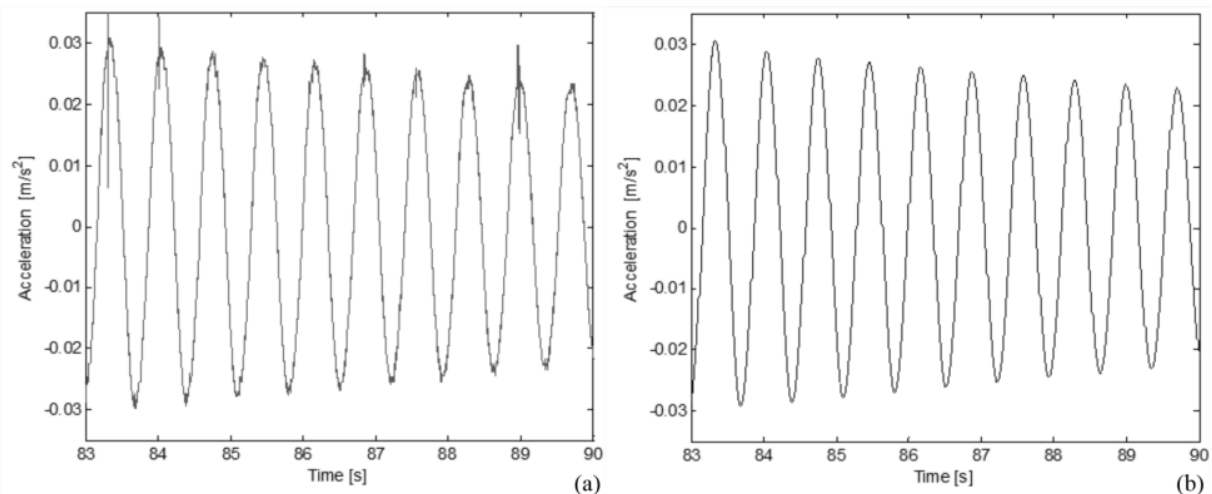

Fig. 7. Detail of acceleration signal (a) before and (b) after filtering for the free decay oscillations episode at a time of 83-200 s.
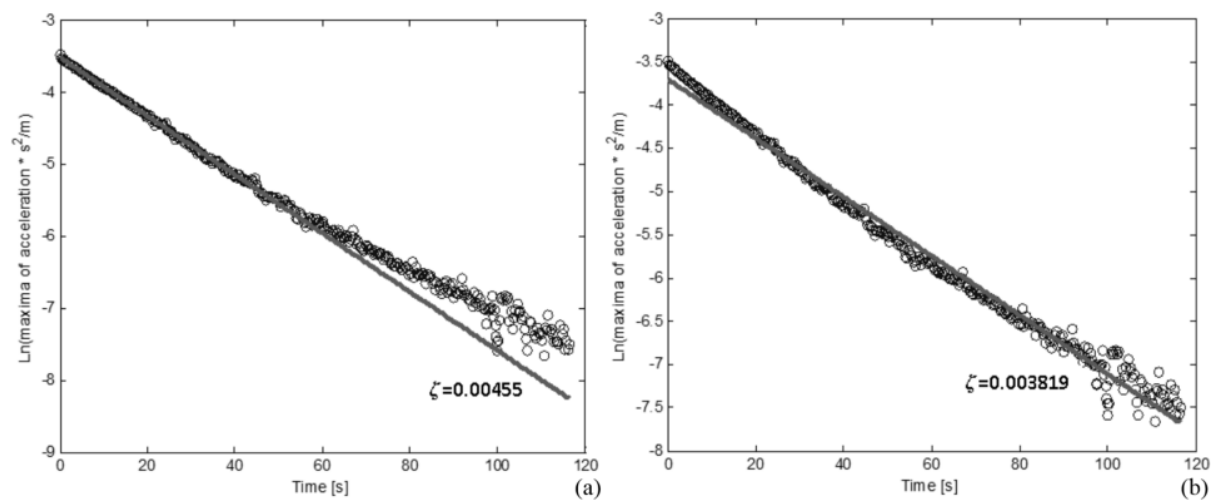

Fig. 8. Linear fit for the free decay oscillations episodes at a time of 83-200 s: (a) using the peaks of the first $20 \%$ of the signal and (b) using all of the peaks.
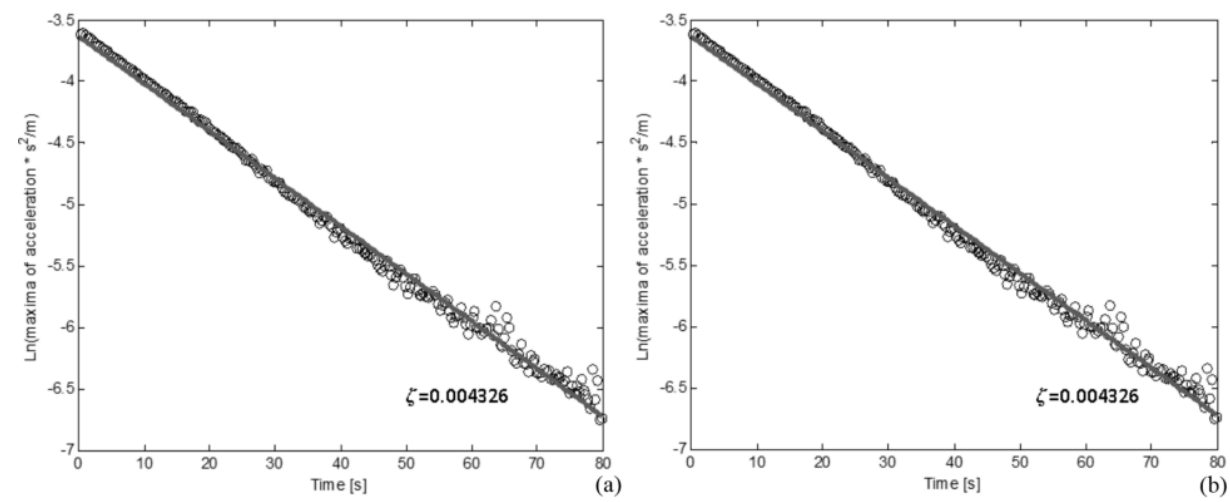

Fig. 9. Linear fit for the free decay oscillation episodes at a time of 537-616 s: (a) using the peaks of the first $20 \%$ of the signal and (b) using all of the peaks. 
Table 1. Damping ratio values from episodes of different free decay oscillations.

\begin{tabular}{|c|c|c|}
\hline Time $[\mathbf{s}]$ & $\begin{array}{c}\zeta[\%] \text { First 20\% } \\
\text { of the peaks }\end{array}$ & $\begin{array}{c}\zeta[\%] \mathbf{1 0 0 \%} \text { of } \\
\text { the peaks }\end{array}$ \\
\hline $7.7-73$ & 0.4561 & 0.4430 \\
\hline $83-200$ & 0.4550 & 0.3819 \\
\hline $273-316$ & 0.4360 & 0.4782 \\
\hline $486-520$ & 0.4360 & 0.4782 \\
\hline $537-616$ & 0.4326 & 0.4326 \\
\hline
\end{tabular}

Under the linearity and decoupling hypotheses assumed herein, the decoupled equations of motion of the sphere along the streamwise, $x$ and transverse, $y$ directions are:

$$
\begin{aligned}
& F_{x}=m \ddot{x}+c \dot{x}+k x \\
& F_{y}=m \ddot{y}+c \dot{y}+k y
\end{aligned}
$$

where $F_{x}$ and $F_{y}$ are the total fluid forces in the streamwise and transverse direction, respectively; $m$ is the total oscillating structural mass (i.e., not including fluid added mass); and $k$ and $c$ are the stiffness and the damping coefficients, respectively, that were previously estimated.

The instantaneous velocities $(\dot{x}, \dot{y})$ and accelerations $(\ddot{x}, \ddot{y})$ in $(3,4)$ were obtained by the Centred Finite Difference Method (CFDM) starting from the streamwise and transverse displacements $(x, y)$ as measured within the experimental campaign described in Section 2.

\section{Discussion of the results}

In this study, we investigated fluid-dynamic loading on an oscillating sphere located at three different relative submergence conditions $h^{*}=2, h^{*}=3.97$ and $h^{*}=0$ in steady free surface flows (Table 2) to analyse how the presence of both the flow free surface and channel bottom influences the fluid-dynamic loading acting on the sphere.

First, we report the dynamic response in terms of amplitude, $A^{*}$, and frequency, $f^{*}$, as related to the three submergence conditions already considered by Mirauda et al. [11-14]. In particular, the experimental condition of $h^{*}=2$ (a completely submerged sphere) is compared with those observed in literature. The dynamic response in the function of the reduced velocity $U^{*}\left(U^{*}=U / f_{n w} D\right)$ is reported in Figure 10. This figure highlights how the trend of oscillation amplitudes is crescent with $U^{*}$, which is similar to the first branch of the literature curve (Fig. 10a) and tends to reach Mode I. Moreover, from the frequency response in Figure 10b, it is possible to observe how the system tends to reach a resonance condition that is typical of the periodic Mode I, in which the oscillation frequency is close to the natural frequency for the higher flow velocity $\left(U^{*}=4.74\right)$.
Table 2. Experimental tests [11].

\begin{tabular}{|c|c|c|}
\hline Test & $\boldsymbol{h}^{*}$ & $\boldsymbol{U}^{*}$ \\
\hline 1 & 2 & 1.90 \\
\hline 2 & 3.97 & 1.90 \\
\hline 3 & 0 & 1.90 \\
\hline 4 & 2 & 2.84 \\
\hline 5 & 3.97 & 2.84 \\
\hline 6 & 0 & 2.84 \\
\hline 7 & 2 & 3.79 \\
\hline 8 & 3.97 & 3.79 \\
\hline 9 & 0 & 3.79 \\
\hline 10 & 2 & 4.74 \\
\hline 11 & 3.97 & 4.74 \\
\hline 12 & 0 & 4.74 \\
\hline
\end{tabular}
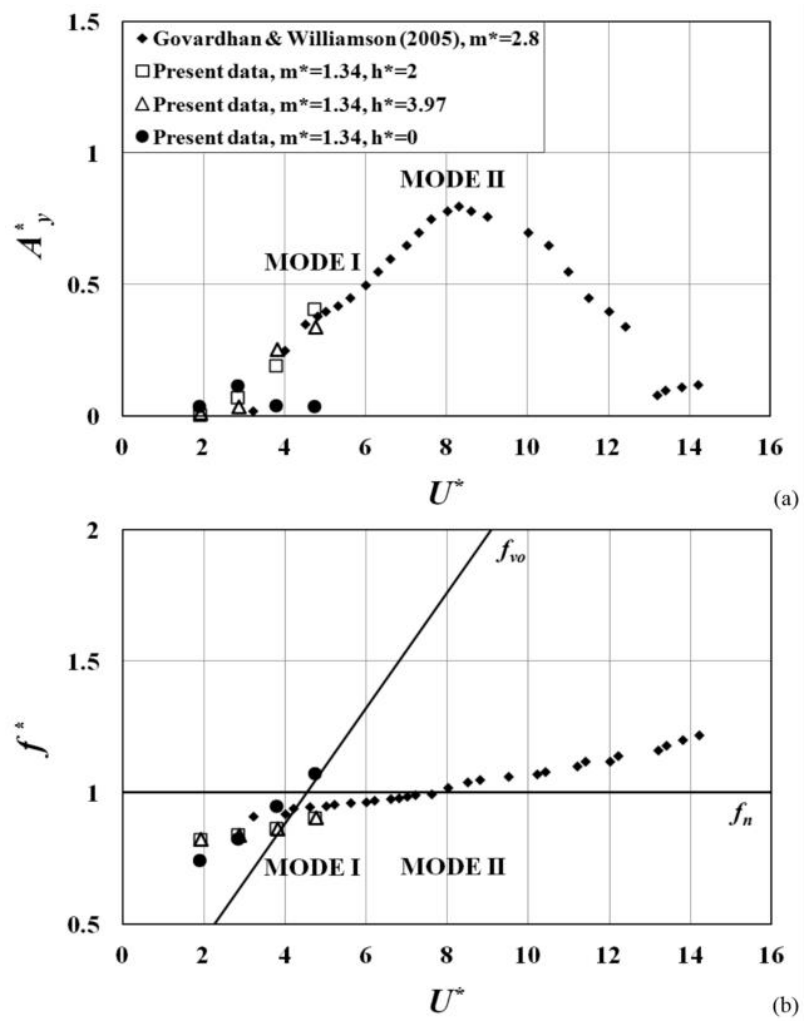

(b)

Fig. 10. (a) Transverse normalised amplitudes $\left(A^{*}\right)$ and (b) frequency ratios $\left(f^{*}\right)$ at different relative submergences $\left(h^{*}\right)$ in the function of $U^{*}$.

The other two conditions, $h^{*}=3.97$ and $h^{*}=0$, were never analysed in the literature and show different behaviours (Fig.10). In detail, for $h^{*}=3.97$, the trend of the oscillation amplitudes and frequencies are similar to those of $h^{*}=2$, which show how the presence of the bottom does not influence the dynamic response of the system. On the contrary and in the case of $h^{*}=0$, a first increase and subsequent decrease of $A^{*}$ for $U^{*}>2.84$ are observed, and the latter was induced by the distortion of the free surface, which inhibits the development of a dynamic response typical of Mode I. The frequencies trend in the case of $h^{*}=0$ is always a crescent but seems to be distant from Mode I (Fig. 10b). Within the experimental range shown in Figure 10, the fluid-

\footnotetext{
*Corresponding author: domenica.mirauda@unibas.it
} 
dynamic loading that was acting on the sphere was calculated with $(3,4)$; moreover, it was also split into a "potential force" component $\left(F_{p}\right)$ and a "vortex force" component $\left(F_{v}\right)$ due to the dynamics of vortices as performed by $[8,16,17]$ and several other studies:

$$
F_{(x, y)}=F_{p(x, y)+} F_{v(x, y)}
$$

where the potential force can be calculated as:

$$
F_{p(x, y)}=-C_{A} m_{d}(\ddot{x}, \ddot{y}) t=-m_{d}(\ddot{x}, \ddot{y}) t
$$

in which $m_{a}$ is the added mass $\left(C_{A} m_{d}\right)$ and $\ddot{x}(t)$ and $\ddot{y}(t)$ are the instantaneous accelerations of the sphere in the streamwise and transverse directions, respectively. Normalising the forces by $1 / 2 \rho U^{2} \pi D^{2} / 4$, the force coefficients $\left(C_{x}\right.$ and $\left.C_{y}\right)$ and potential components $\left(C_{p}\right)$ can be computed, whereas the vortex force coefficients $\left(C_{v}\right)$ were computed as the difference between the total and potential components.

When the experimental results evidence the resonance condition of Mode I, we also considered the hypothesis of sinusoidal behaviour to solve the equation of motion as reported by [8]. In most cases of vortexinduced vibrations, the frequency of body oscillations are synchronised with the periodic vortex wake mode downstream of the body, and therefore, the transverse amplitude response $y(t)$ and the fluid force $F_{y}(t)$ are well represented by:

$$
\begin{gathered}
y(t)=-A \sin (2 \pi f t) \\
F_{y}(t)=F_{0} \sin (2 \pi f t+\phi)
\end{gathered}
$$

where $f$ is the transverse oscillation frequency $\left(f_{y}\right)$ and $\varphi$ is the phase angle between the total fluid force and the transverse body displacement. Therefore, the solution of Eq. (4) is:

$$
A^{*}{ }_{y}=y / D=3 / 32 \pi^{2} \quad C_{y} \sin \phi /\left(m^{*}+C_{A}\right) \zeta_{w}\left(U^{*} / f^{*}\right)^{2} f^{*}
$$

where:

$$
f^{*}=f / f_{n w}=\left[\left(m^{*}+C_{A}\right) /\left(m^{*}+C_{E A}\right)\right]^{0.5}
$$

and $C_{E A}$ is an effective added mass coefficient that includes an apparent effect due to the transverse total fluid force in phase with the body acceleration $\left(C_{y} \cos \varphi\right)$ :

$$
C_{E A}=3 / 16 \pi^{2} A^{*}{ }^{*}\left(U^{*} / f^{*}\right)^{2} C_{y} \cos \phi
$$

By combining these three equations, we computed the transverse force coefficient $\left(C_{y}\right)$ acting on the sphere when knowing the transverse displacements, $y$, and oscillation frequency, $f_{y}$.

Figures 11 and 12 report the transverse displacements $(y / D)$ normalised with respect to the sphere diameter and coefficient $\left(C_{y}\right)$ compared for each different $h^{*}$; moreover, we report the transverse coefficients split into the potential $\left(C_{p, y}\right)$ and vortex $\left(C_{v, y}\right)$ components, as discussed before. The figures refer to Tests 1, 2, 3 (Figure 11), 10, 11 and 12 (Fig. 12) of Table 2. The mean component of the displacements and force coefficients remain constant around the zero value due to the symmetry of the set-up. The comparison between Figures 11 and 12 highlight the increase of the displacement and force fluctuating components with the increasing velocity when the sphere is far from the free surface $\left(h^{*}=2\right.$ and $\left.h^{*}=3.97\right)$. Instead, and different from these two submergence conditions, a reduction of the displacement and force fluctuating components occurs at $h^{*}=0$ with the increase of the reduced velocity (Figs. 11a, 11b, 12a and 12b). In the case of the forces, the splitting of the transverse coefficient into its potential $\left(C_{p, y}\right)$ and vortex $\left(C_{v, y}\right)$ components highlights that this behaviour is imputable to the vortex component of the transverse force coefficient.


Fig. 11. (a) Trend of normalised transverse oscillations $(y / D)$; (b) transverse total force $C_{y}$; and (c) and (d) are the potential and vortex component of the transverse total force for low flow velocity $\left(U^{*}=1.90\right)$. 

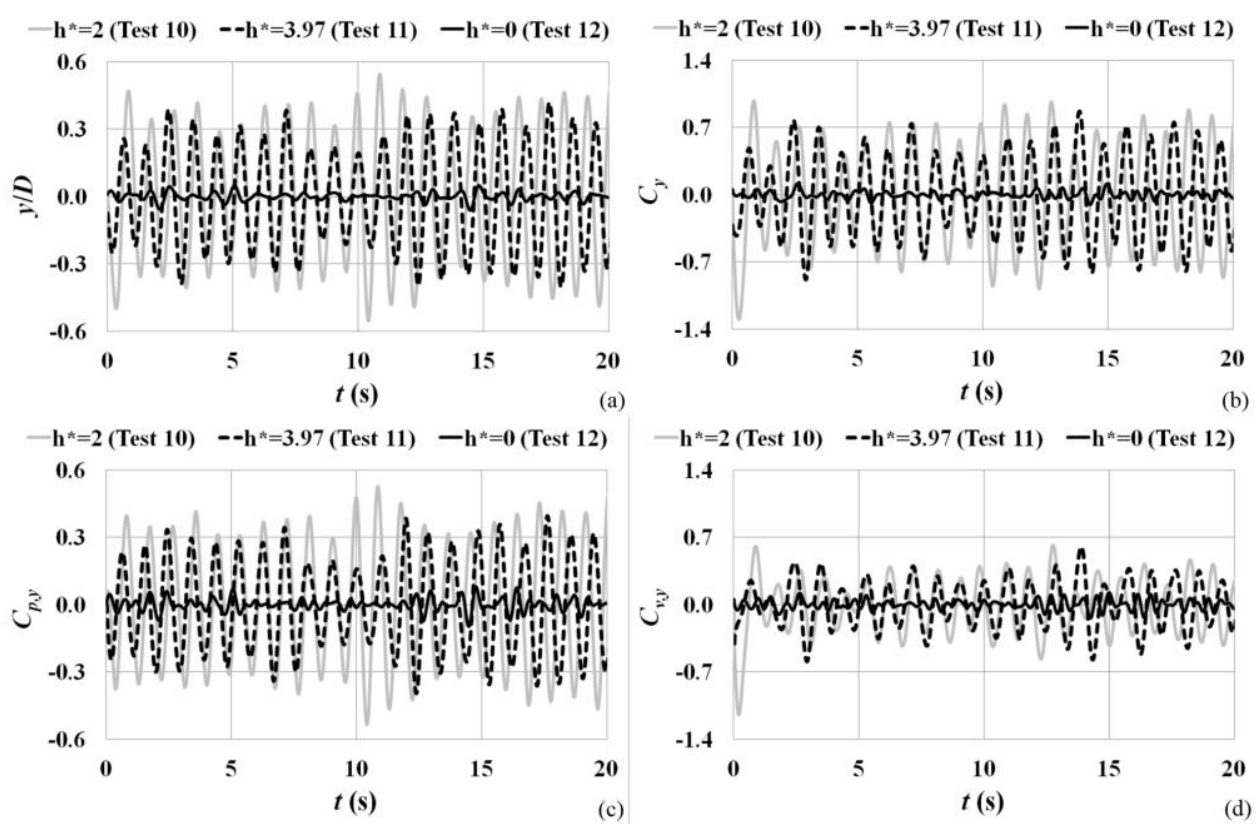

Fig. 12. (a) Trend of normalised transverse oscillations $(y / D)$; (b) transverse total force $C_{y}$; and (c) and (d) are the potential and vortex component of the transverse total force for high flow velocity $\left(U^{*}=4.74\right)$.

The experimental data lead us to consider that the strong distortion of the free surface, which occurs when $h^{*}=0$ and the flow velocity is larger than $U^{*}=2.84$, noticeably reduces the fluctuating component of both the displacements and transverse force coefficient acting on the sphere. By contrast, for values of $U^{*}<2.84$, we observe an increase of the transverse displacement fluctuating component and a significant influence of the vortex component on the transverse force coefficient. As discussed before, when Mode I is achieved, the transverse force coefficient $\left(C_{y}\right)$ acting on the sphere could be computed by knowing the transverse displacements, $y$, and oscillation frequency, $f_{y}$, under the hypothesis of sinusoidal behaviour. With the aim of verifying the force coefficient evaluation, we compared the methods discussed before. We considered the case of $U^{*}=4.74$ with $h^{*}=2$ and $h^{*}=3.97$; which are conditions when the sinusoidal behaviour is prevalent in the transverse displacements (Figs. 13a-f) and Mode I is achieved (Fig. 10). Instead, we could not consider the condition of $h^{*}=0$ for the low periodicity of the signal as observed in Figures $13 \mathrm{~g}-\mathrm{i}$, probably due to the strong deformation of the free surface above the sphere that influences body movement and induces random motion.


Fig. 13. Power spectra of the transverse force coefficients at (a), (b), and (c) $h^{*}=2$; (d), (e), and (f) $h^{*}=3.97$; and (g), (h), and (i) $h^{*}=0$ for high flow velocity $\left(U^{*}=4.74\right)$. 
Figures 14 and 15 report the trends of the force coefficients derived from the computation of the body instantaneous velocities and accelerations by means of the CFDM (black line). These trends also come from the solution of the motion equation according to [8] (grey line) for the conditions of relative submergence $h^{*=} 2$ and $h^{*}=3.97$ and for the higher flow velocity $\left(U^{*}=\right.$ 4.74, Test 10 and 11). For this velocity of the flow, the sphere is close to the resonance condition of Mode I. The analysis of the curves shows how the results are, in the resonance situation, very similar.


Fig. 14. (a) Transverse total force $C_{y}$ and (b) and (c) are the potential and vortex component, respectively, of the transverse total force for high flow velocity $\left(U^{*}=4.74\right)$ at $h^{*}=2$ (Test $10)$.

Instead, Figures $16 \mathrm{a}$ and $16 \mathrm{~b}$ report that the behaviour of the streamwise movement was normalised with respect to the sphere diameter and the drag coefficients for the three relative submergences $h^{*}$ at a low flow velocity $\left(U^{*}=1.90\right.$; Test 1,2 and 3$)$. It is important to note how the mean and fluctuating components of the drag coefficient are significantly greater in the asymmetrical boundary conditions $(h *=0$ and $h^{*}=3.97$ ).

In addition, Figures $16 \mathrm{c}$ and $16 \mathrm{~d}$ show that the drag coefficients split into the potential $\left(C_{p, x}\right)$ and vortex $\left(C_{v, x}\right)$ components, as previously discussed. As expected, the potential component of drag (Fig. 16c) moves around zero, whereas the vortex component (Fig. 16d) contains the mean and part of the fluctuating components of the drag coefficient.
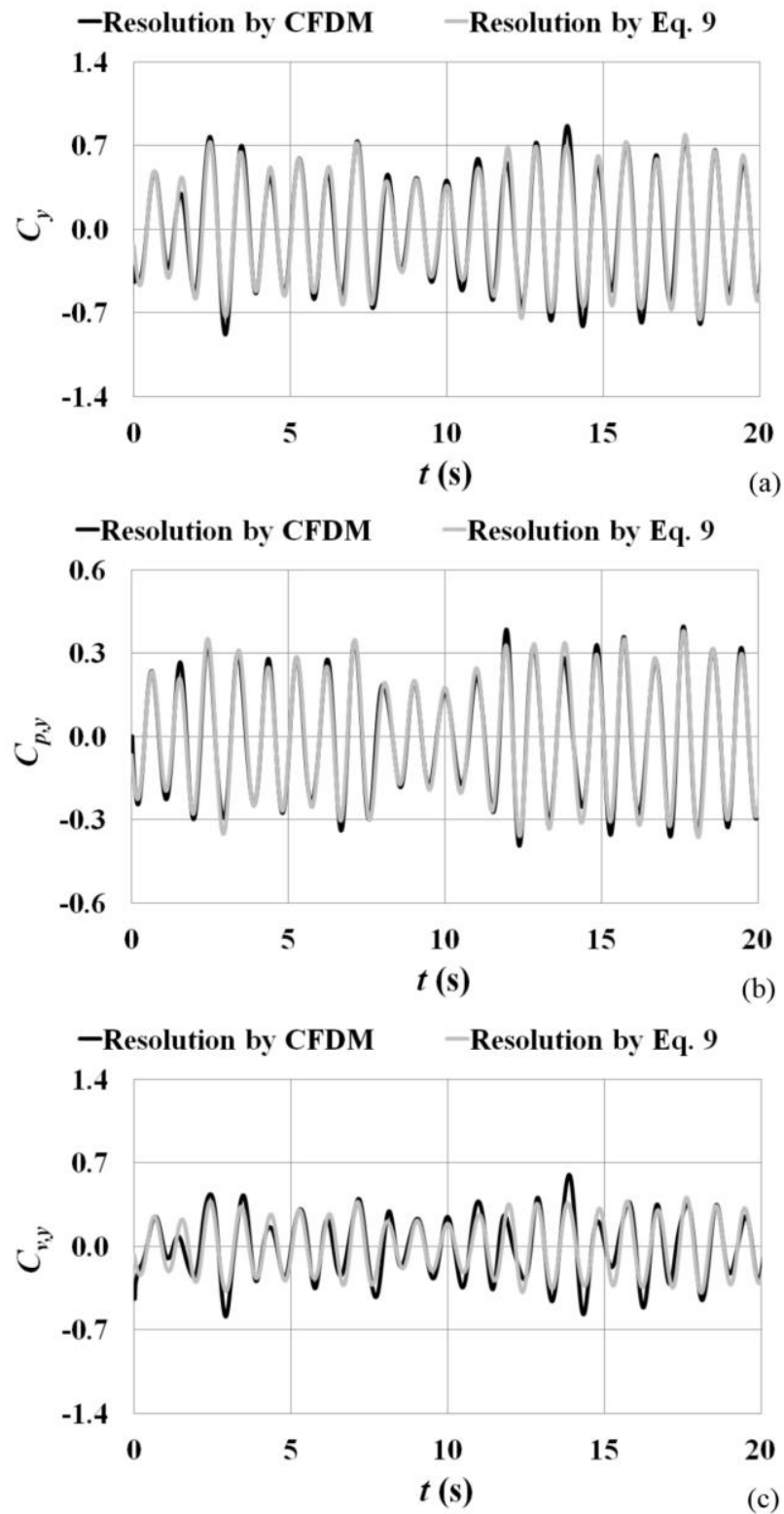

Fig. 15. (a) Transverse total force $C_{y}$ and (b) and (c) are the potential and vortex component, respectively, of the transverse total force for high flow velocity $\left(U^{*}=4.74\right)$ at $h^{*}=3.97$ (Test $11)$.

In addition to, as it is possible to note through the power spectra of Figure 17, in the cases of $h^{*}=3.97$ and $h^{*}=0$, the signal is periodic with one predominant peak of approximately $1 \mathrm{~Hz}$, whereas under the condition of $h^{*}=2$, an overlapping of non-periodic signals is observed

* Corresponding author: domenica.mirauda@unibas.it 
with a range of frequencies between 0.7 and $1.5 \mathrm{~Hz}$. By increasing the velocity from $U^{*}=1.90$ to $U^{*}=4.74$ (Fig. 18a), the mean displacements of the sphere in the $x$ direction, with respect to its static position (zero value), are amplified. However, for this higher flow velocity (Fig. 18), interestingly enough, the results are different in terms of the mean and fluctuating components of the force coefficients along the $x$ direction. As Figure $18 \mathrm{~b}$ depicts, the mean values of $C_{x}$ follow the ordering displayed in Figure 18a for the displacements. For $h^{*=}$ 2 , an increase of the mean value of $C_{x}$ is noted as well as of the fluctuating component, whereas the case of $h^{*}=$ 3.97 also leads to a larger mean value associated with a larger loss of periodicity. Moreover, $h^{*}=0$ leads to the lowest value of $C_{x}$ (the mean value of $C_{x}$ is 1.25 while the standard deviation is $s t d C_{x}=0.05$ for $U^{*}=4.74$ ), which is a value lower than that found for $U^{*}=1.90$ $\left(C_{x}=1.47\right.$ and $\left.s t d C_{x}=0.43\right)$. In this case, the amplitude of the fluctuating part is also reduced, which suggests a radical change in the mechanism of the vortex detachment.

Such behaviour could be due to the presence of a jetlike flow between the upper surface of the sphere and free surface, which reduces both the mean and fluctuating component of the forces.
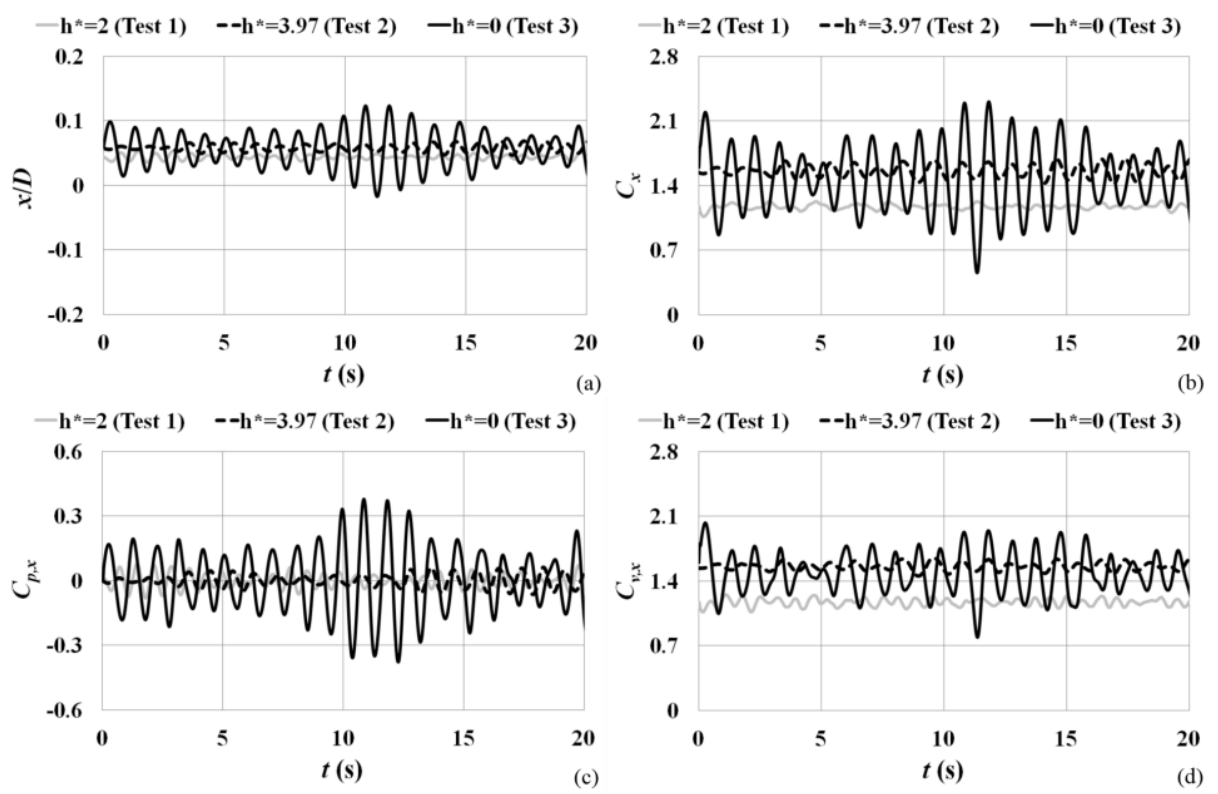

Fig. 16. (a) Trend of normalised streamwise oscillations $(x / D)$; (b) streamwise total force $C_{x}$; and (c) and (d) are the potential and vortex component, respectively, of the streamwise total force for low flow velocity $\left(U^{*}=1.90\right)$.
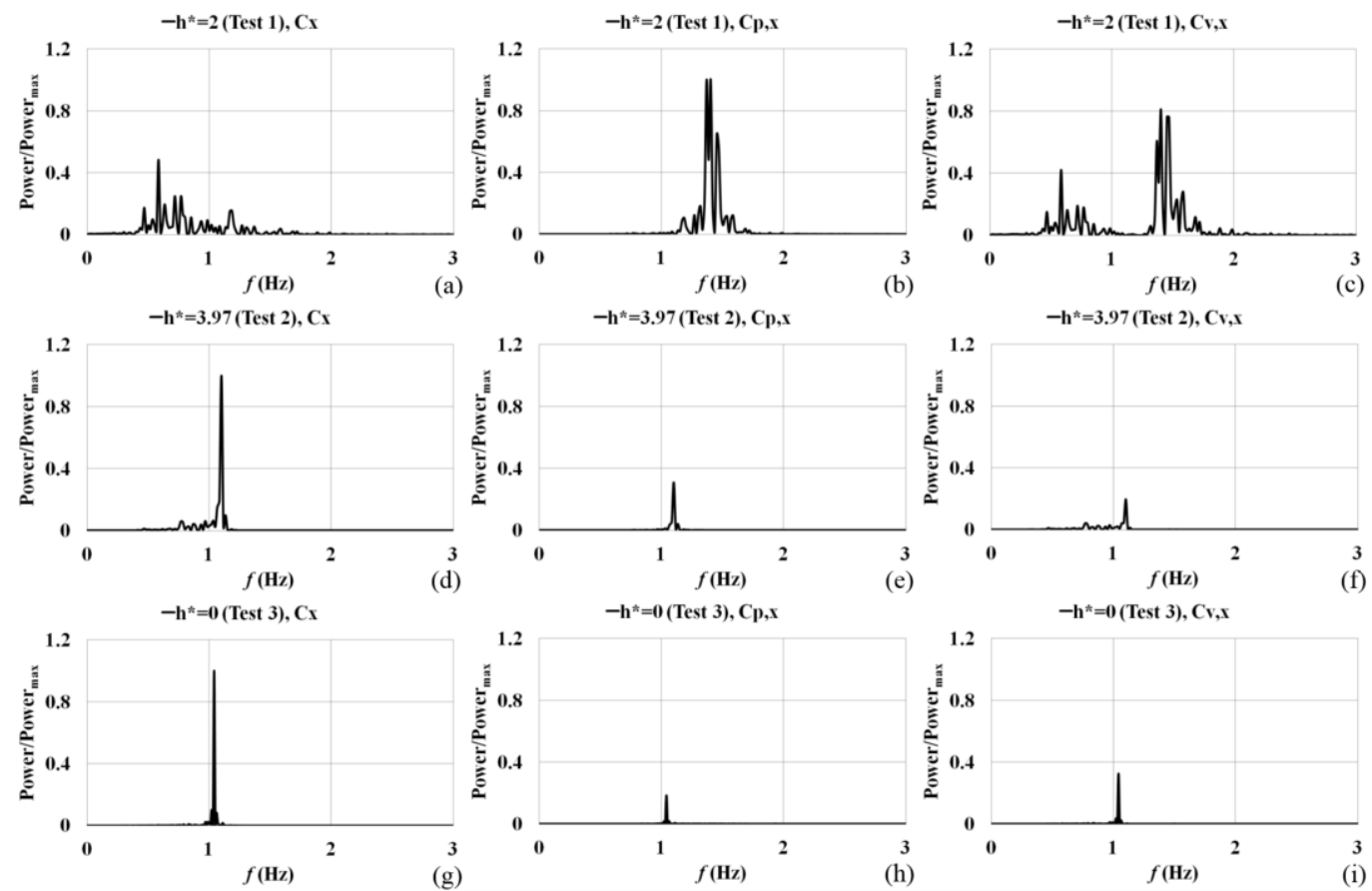

Fig. 17. Power spectra of the streamwise force coefficients at (a), (b), and (c) $h^{*}=2$; (d), (e), and (f) $h^{*}=3.97$ and (g), (h), and (i) $h^{*}=0$ for low flow velocity $\left(U^{*}=1.90\right)$. 



Fig. 18. (a) Trend of normalised streamwise oscillations $(x / D)$; (b) streamwise total force $C_{x}$; and (c) and (d) are the potential and vortex component, respectively, of the streamwise total force for high flow velocity $\left(U^{*}=4.74\right)$.

\section{Conclusions}

The measurements of the forces acting on the structure with simplified geometry described in this paper represent a necessary step for the numerical resolution of coupled mechanical-fluid dynamic models. Knowledge of the internal forces in the mechanical model permits computation of the fluid dynamics from its motion. The internal forces were reconstructed after establishing a correlation between the internal forces and motion of the mechanical model.

The results underlined an increase of the mean components of the force coefficients along the streamwise direction in the asymmetrical conditions of flow for low velocity with respect to the condition of $h^{*}=2$. Instead, a reduction of the mean component is observed for the higher velocity in the case of $h^{*}=0$. Such behaviour is explained by the presence of a free surface distortion, which reduces the mean component of the force. Instead, the fluctuating components show similar values for all of the conditions. Only in the case of $h^{*}=0$, at the investigated low velocity, is it possible to observe larger values of displacement and force coefficients due to the presence of a jet-like flow between the upper surface of the sphere and free surface, which increases the movements and forces acting on the sphere.

Along the transverse direction and for a low velocity, it is interesting to note a behaviour of the system similar to the case of the streamwise direction with higher fluctuations of displacements and forces for $h^{*}=0$, whereas for high velocities, it evolves in the opposite manner. In fact, for $h^{*}=0$, the strong distortion of the free surface above the sphere reduces the displacements and force fluctuating components and influences the body to induce a random movement. In the case of $h^{*}=$ 3.97 and $h^{*}=2$, we instead note a considerable increase of fluctuating components and a repeatable trend with sinusoidal functions, which is typical of the resonance condition already observed in the literature by several authors.

Finally, an additional interesting result is the significant values assumed by the vortex fluid force, which as discussed, could provide an important contribution to the analysis of this type of phenomenon.

\section{References}

1. J.M. Jonkman, P.D. Sclavounos, Development of fully coupled aeroelastic and hydrodynamic models for offshore wind turbines, 44th AIAA Conference: Aerospace Sciences Meeting and Exhibit Online Proceedings, Reno, Nevada, 10-12 January (2006)

2. A. Amicarelli, R. Albano, D. Mirauda, G. Agate, A. Sole, R. Guandalini, A smoothed particle hydrodynamics model 3D solid body transport in free surface flows, Computers \& Fluids, 116, 205 228 (2015)

3. R. Albano, A. Sole, D. Mirauda, J. Adamowski, Modelling large floating bodies in urban area flashfloods via a smoothed particle hydrodynamics model, J. of Hydrology, 541, 344-358 (2016)

4. S.K. Chakrabarti, Hydrodynamic of offshore structures, Springer-Verlag, New York (1987)

5. M. Brankovic, P.W. Bearman, Measurements of transverse forces on circular cylinders undergoing vortex-induced vibration, J. Fluids and Struct. 22, 829-836 (2006)

6. T.L. Morse, C.H.K. Williamson, Employing controlled vibrations to predict fluid forces on a 
cylinder undergoing vortex-induced vibration, J. Fluids and Struct. 22, 877-884 (2006)

7. C.H.K. Williamson, R. Govardhan, A brief review of recent results in vortex-induced vibrations, J. Wind Eng. and Ind. Aerodyn. 96, 713-735 (2008)

8. R. Govardhan, C.H.K. Williamson, Vortex induced vibration of a sphere, J. Fluid Mech. 531, 11-47 (2005)

9. R. Govardhan, C.H.K. Williamson, Vortex induced motions of a tethered sphere, J. Wind Eng. and Ind. Aerodyn. 69-71, 375-385 (1997)

10. C.H.K. Williamson, R. Govardhan, Dynamics and forcing of a tethered sphere in a fluid flow, J. Fluids and Struct. 11, 293-305 (1997)

11. D. Mirauda, A. Volpe Plantamura, S. Malavasi, Hydrodynamic forces acting on an oscillating structure, Proc. 2nd International Conference on Physical Coastal Processes, Management and Engineering, Coastal Processes 2011, WIT Transactions on Ecology and Environment, WIT Press, Naples, Italy 149, 321-331 (2011)

12. D. Mirauda, A. Volpe Plantamura, S. Malavasi, Boundaries effects on the movements of a sphere immersed in a free surface flow, J. Offshore Mech. and Arct. Eng. 133, 041301-1-041301-5 (2011)

13. D. Mirauda, A. Volpe Plantamura, S. Malavasi, Boundaries influence on the flow field around an oscillating sphere, Proceedings of the ASME 2013 32nd International Conference on Ocean, Offshore and Arctic Engineering OMAE2013, June 9-14, Nantes, France (2013)

14. D. Mirauda, A. Volpe Plantamura, S. Malavasi, Dynamic response of a sphere immersed in a shallow water flow, J. Offshore Mech. and Arct. Eng. 136, 021101-1-021101-6 (2014)

15. P.D. Welch, The Use of Fast Fourier Transform for the Estimation of Power Spectra: A Method Based on Time Averaging Over Short, Modified Periodograms, IEEE Trans. Audio Electroacust. AU-15, 70-73 (1967)

16. R. Govardhan, C.H.K. Williamson, Modes of vortex formation and frequency response of a freely vibrating cylinder, J. Fluid Mech. 420, 85-130 (2000)

17. J. Lightill, Wave loading on offshore structures, J. Fluid Mech. 173, 667-681 (1986) 